\title{
Jürgen Wolters
}

\author{
Uwe Hassler ${ }^{1}$
}

Online publiziert: 15 . Februar 2016

(C) Springer-Verlag Berlin Heidelberg 2016

Wie hoch von nöten sey Arithmetic/

und die gantze Mathematische Kunst/

kann man hieraus leichtlich ermessen/

daß nichts bestehen mag/

so nicht mit gewisser Zahl und Maß vereint.

Adam Risen, Rechenbuch, 1574

(Zitiert aus dem Vorwort von Heinz König und Jürgen Wolters, Einführung in die Spektralanalyse ökonomischer Zeitreihen, 1972, Verlag Anton Hain.)

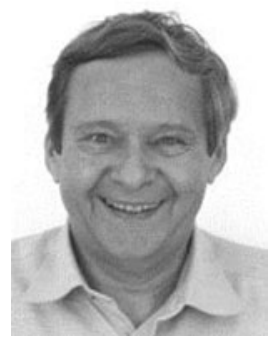

Foto: Thomas Ausserhofer

Am 21. November 2015 verstarb Prof. Dr. Jürgen Wolters im Alter von 75 Jahren in Berlin. Sein Tod kam völlig unerwartet. Im Sommer 2015 war er mit seiner Frau Heide in Neuseeland gewesen, wo er an der University of Canterbury einen Doktorandenkurs gegeben hatte. Noch im Herbst des letzten Jahres schmiedete er mit dem Autor dieser Zeilen Pläne zur Neuauflage eines Buches, und für Anfang Dezember hatte er einen Flug nach Kiew gebucht, wo er einen Kurs unterrichten wollte. Er hinterlässt eine große Lücke nicht nur in der empirischen Wirtschaftsforschung.

Jürgen Wolters kam am 24. Juni 1940 in Stuttgart zur Welt. Er verbrachte dort seine Kindheit und Jugend. Auch während seines Studiums der Mathematik, das er 1966 mit einem Diplom abschloss, blieb er seiner Heimatstadt treu. Danach ging er an die Universität Mannheim, wo er 1972 unter der Betreuung von Heinz König mit der Arbeit „Spektralanalytische Schätzung linearer dynamischer Systeme“ promoviert wurde. Diese 1973 publizierte Dissertation wurde mit dem IHK-Preis für wissenschaftliche Arbeiten ausgezeichnet. Zumindest im deutschsprachigen Raum

Uwe Hassler

hassler@wiwi.uni-frankfurt.de

$1 \quad$ Frankfurt am Main, Deutschland 
darf Jürgen Wolters als einer der Pioniere der Spektralanalyse in der Ökonometrie betrachtet werden. Auch seine Habilitationsschrift ,Stochastic Dynamic Properties of Linear Econometric Models" war modernen Methoden und Anwendungen der Zeitreihenökonometrie gewidmet und brachte ihm 1978 von der Universität Mannheim die venia legendi in Volkswirtschaftslehre und Ökonometrie ein; die Arbeit wurde 1980 im Springer-Verlag publiziert. 1982 nahm er den Ruf auf eine Professur am Institut für Statistik und Ökonometrie des Fachbereichs Wirtschaftswissenschaft der Freien Universität Berlin an, die er bis zu seinem Ruhestand im Jahr 2008 innehatte. Allerdings waren sein Tatendrang und seine Aktivitäten in Lehre und Forschung auch nach 2008 ungebrochen.

Im Bereich Ökonometrie/Angewandte Statistik trug Jürgen Wolters auf vielfache Weise zum wissenschaftlichen Fortschritt nicht nur in Deutschland bei. Er publizierte zahlreiche Artikel in international einschlägigen Zeitschriften und Bücher mit Koautoren aus dem In- und Ausland. Sein Forschungsschwerpunkt blieb die Anwendung von Zeitreihenanalyse auf ökonomische Fragestellungen. Dabei zeichnet es seine Arbeiten aus, an der Schnittstelle von aktueller statistischer Methode und ökonomisch relevanter Anwendung angesiedelt zu sein. Konsequenterweise war er gleichermaßen in der Deutschen Statistischen Gesellschaft wie im Verein für Socialpolitik engagiert. In Letzteren wurde er 1979 aufgenommen und stand dort dem Ökonometrieausschuss von 1988 bis 1991 vor. Im Folgejahr 1980 trat Jürgen Wolters der Deutschen Statistischen Gesellschaft bei und übernahm den Vorsitz des Ausschusses für Empirische Wirtschaftsforschung und Angewandte Ökonometrie von 1999 bis 2003.

Jürgen Wolters war in hohem Maße an Zusammenarbeit interessiert, er konnte inspirieren und war offen für Inspiration. Er reiste nicht nur gern zu Forschungs- und Lehrzwecken ins Ausland, sondern war auch ein herzlicher Gastgeber in Berlin, und entsprechend fruchtbar waren seine nationalen und internationalen Kooperationen. Gleichzeitig besaß er auch Talent zu Führung, Beratung und Gestaltung. So war er über viele Jahre Direktor der Volkswirtschaftlichen Abteilung an der FU Berlin, Vorsitzender des Promotionsausschusses und leitete ein Teilprojekt des Sonderforschungsbereichs 373 der Humboldt-Universität zu Berlin von 1994 an über 10 Jahre. Er war von 1998 bis 2006 Mitglied im Kuratorium des DIW Berlin und dort Forschungsdirektor für Makroökonometrie von 2000 bis 2005. Überdies gehörte er dem Stiftungsrat Geld und Währung der Deutschen Bundesbank an. So kann es nicht wundern, dass viele seiner empirischen Arbeiten Anwendungen aus dem Bereich der Geldpolitik gewidmet sind. Besondere Schwerpunkte lagen dabei auf Studien zu Zinsstruktur und internationalem Zinszusammenhang, zur Stabilität der Geldnachfrage und zum geldpolitischen Transmissionsmechanismus.

Als Professor fühlte sich Jürgen Wolters auch besonders dem wissenschaftlichen Nachwuchs verpflichtet. Als fördernder und fordernder Lehrer hat er Hunderten von Studierenden empirisches Arbeiten nahe gebracht. Schon 1988, also zu einer Zeit als strukturierte Doktorandenprogramme an wirtschaftswissenschaftlichen Fachbereichen in Deutschland eine Rarität waren, machte er sich maßgeblich um das Graduiertenkolleg „Angewandte Mikroökonomik“ an der FU Berlin verdient. In großer Zahl prägte er Doktorandinnen und Doktoranden, die heute vielfach einflussreiche Positionen innehaben. 
Der Autor dieser Zeilen lernte Professor Wolters 1985 als Student im Grundstudium kennen. Über 30 Jahre durfte ich von ihm lernen, später mit ihm lehren und publizieren. In dieser Zeit traf ich sehr viele Menschen, die Jürgen Wolters ausgesprochen dankbar und sehr zugetan waren und sind. Mit seinem Optimismus und Enthusiasmus machte er unzähligen jungen Menschen Mut; seine vorurteilsfreie Offenheit und Neugier, sein Interesse, haben uns beflügelt; auf seine großzügige und kollegiale Unterstützung war immer Verlass. Ansteckend waren seine Lebensfreude, sein Spaß am Sport, der ihn bis zuletzt Tennis spielen ließ, seine Vielseitigkeit und seine Unternehmungslust. Gleichzeitig zeigte uns Jürgen Wolters mit seiner Bescheidenheit und trotz seiner Leidenschaft für den Beruf, dass Erfolg nicht alles ist. Er selbst ließ nie einen Zweifel daran, dass seine Frau, seine beiden Söhne und seine Enkel für ihn das Kostbarste im Leben waren.

Am 4. Dezember 2015 wurde Jürgen Wolters auf dem Waldfriedhof Dahlem in Berlin im Kreis seiner Familie, vieler Kollegen, Schüler und Freunde in großer Trauer beigesetzt. 